\title{
EYPETHPIO ГYГГРАФЕ AUTHOR INDEX
}

Adamaki A.K.: 1984

Agalos A.: 2005

Aidona E.: 1888

Albanakis K.: 2383

Alexandratos V.G.: 2310

Alexandri M.: 1056

Alexandropoulou S.: 989

Alexandrou M.: 1888

Alexopoulos A.: 1792

Alexopoulos J.D.: 1898

Alexouli-Livaditi A.: 737

Alkalais E.: 1286

Amerikanos P.: 1149

Anagnostou Ch.: 2426

Anagnostoudi Th.: 548

Angelopoulos A.: 1094

Antonakos A.: 1821

Antonarakou A.: 568, 613, 620, 763

Antonelou A.: 876

Antoniou A.A.: 1104

Antoniou Var.: 320

Antoniou Vas.: 320

Apostolaki Ch.: 2570

Apostolidis Em.: 1418, 1619, 1850

Apostolidis N.: 2532

Apostolidis N.: 2597

Arapogiannis E.: 2229

Argyraki A.: 1737, 2319, 2510

Argyriadis I.: 264

Arvanitidis N.D.: 2437

Arvanitis A.A.: 1907, 2246

Astiopoulos A.C.: 1994

Athanassouli E.: 939

Avgerinas A.: 276

Avramidis P.: 558, 654

Ballas D.: 1056, 1737

Baltzois V.: 1149

Bantekas I.: 829

Barbera G.: 663

Barbu O.: 594

Baskoutas I.: 2125

Bathrellos D.G.: 1637

Bathrellos G.D.: 1572

Batsalas A.: 697

Baziotis I.: 2485, 2522, 2667

Behrends T.: 2310

Bel-lan A.B.: 2338

Bellas M.: 1619
Bellas S.: 579

Beshku H.: 1777

Birke M.: 2338, 2350

Bizoura A.: 1314

Bloukas S.: 1149

Bonsall T.A.: 2406

Bourliva A.: 2532

Bourouni P.: 2540

Brachou C.: 907

Brauer R.: 1267

Brusca L.: 2327

Burgess W.: 1716

Caputo R.: 400, 486

Carey S.: 1056

Catalano S.: 400

Chailas S.: 1919

Chalkias D.: 1335

Charalambopoulos S.: 1878

Chatzaras V.: 387

Chatziangelou M.: 1112

Chatzipanagis I.: 2702

Chatzipetros Al.: 486, 1131, 1383

Chiotis E.: 1539, 1549

Chousianitis K.G.: 1572, 2005

Christanis K.: 224, 2218

Christaras B.: 1112, 1122, 1131, 1267,1672

Christidis G.E.: 2553, 2562, 2570

Christofides G.: 2680

Christoforidou P.: 1678

Çina A.: 2577

Codrea V.: 594

D’ Alessandro W.: 2327

Daftsis E.: 1737

Dasaklis S.: 737

De Vos W.: 2350

Delagrammatikas G.: 2485

Delimani P.: 1074

Demetriades A.: 2338, 2350

Depountis N.: 1138,1210

Dermitzakis M.D.: 86, 978

Diakakis M.: 1323

Diamantis I.: 1697

Diasakos N.: 1149

Dilalos S.: 1898

Dimitrakopoulos D.: 1688

Dimitriou D.: 2229

Dimiza M.D.: 602, 763
Dominic Fortes A.: 2726

Dotsika E.: 886, 958, 1840, 2265, 2383

Doutsou I.: 1350

Doveri M.: 1840

Drakatos G.: 1994

Drinia H.: 613, 620, 763

Dunkl I.: 276

Duris M.: 2338

Economou G.: 804, 2485

Economou N.: 1802

Eikamp H.: 918

Epitropou N.: 939

EuroGeoSurveys Geochemistry Expert Group: 2338, 2350

Exioglou D.: 1230

Fadda S.: 2446, 2588

Fakiris E.: 1064

Falalakis G.: 276

Fassoulas C.: 746

Fassoulas C.: 781, 896, 918

Ferentinos G.: 176, 1018, 1064

Fermeli G.: 978, 989

Fernandez-Turiel J.L.: 2373

Fikos I.: 1953

Filippidis A.: 2373, 2532, 2597, 2762

Filippidis S.: 2597

Fiori M.: 2446, 2588

Foscolos A.E.: 8, 2294

Fotopoulou M.: 2218

Foumelis M.: 1301

Foundas P.: 989

Fountoulis I.: 1046

Frisch W.: 276

Gaki-Papanastassiou K.: 409, 418, 506

Galanakis D.: 1428, 1465

Galbenis C.T.: 2485

Ganas A.: 1607

Garver J.I.: 309

Gawlick H-J.: 276

Georgakopoulos A.N.: 1230, 2236, 2274

Georgiadis I.K.: 2606

Georgiadis P.: 1406

Georgiou A.: 2492

Georgiou Ch.: 1428 
Georgiou P.: 1056

Georgoulas A.: 1074

Geraga M.: 1018, 1064

Germenis N.: 989

Gerogianni N.: 2786

Gerolymatou E.: 1438

Gialamas J.: 1777

Giannakopoulos A.: 958

Giannoulopoulos P.: 1438, 1447

Gimeno D.: 2373

Gioti Ev.: 1627

Gkadri E.: 548

Gkiolas A.: 1272

Gkiougkis I.: 1697

Golubović Deliganni M.: 1582

Gospodinov D.: 1994

Gournelos Th.: 1335, 1647

Hademenos V.: 1539

Hagiou E.: 1157

Haidarlis M.: 907

Hamdan H.: 1802

Handler R.: 299

Hatzipanagiotou K.: 876, 2501, $2540,2617,2712$

Helly B.: 845

Iatrou M.: 1018

Ilia I.: 1590,1688

Iliopoulos G.: 746, 781, 918

İnaner H.: 2218

Ioakim Chr.: 1035

Ioannidis N.: 1888

Janikian Z.: 939

Jenkyns H.C.: 627

Jipa-Murzea C.: 594

Kacandes G.: 2562

Kadetova A.V.: 1341

Kafkala I.G.: 2390

Kafousia N.: 627

Kalantzi F.: 1350

Kalisperi D.: 654

Kallergis G.: 1821

Kallioras A.: 69, 1697

Kalogerogiannis G.: 1149

Kamberis E.: 289, 715

Kanaris D.: 1202, 1230

Kantiranis N.: 2762

Kapetanidis V.: 2015

Karageorgiou D.E.: 1457, 1601, 2229, 2236, 2274, 2692

Karageorgiou M.M.D.: 1601, 2236, 2274

Karagianni A.: 1165

Karagianni E.: 495

Karakaisis G.F.: 46, 2026

Karakitsios V.: 627, 634, 663

Karakonstantis A.: 2043
Karakostas V.G.: 1984, 1994, 2053 , 2064, 2075, 2093, 2114

Karalemas N.: 1707

Karamanos Ch.K.: 2053, 2075

Karapanos E.: 1716

Karastathis V.K.: 1438

Karfakis J.: 1619

Kargiotis E.: 2257

Karipi S.: 2617, 2712

Karmis P.D.: 1393, 1438, 1447, 1919

Karoutzos G.: 1165

Karydakis Gr.: 2246, 2265

Karymbalis E.: 409, 418, 1601

Kastanioti G.: 2786

Kastanis N.: 169

Katagas Ch.: 247

Kati M.: 2786

Katrivanos D.E.: 999

Katsanou K.: 1726, 1878, 2218

Katsiki P.: 2562

Katsikis J.: 2692

Katsonopoulou D.: 812

Kaviris G.: 2084

Kelepertsis A.: 1858

Kelepertzis E.: 1737

Kementzetzidou D. A.: 2053

Keupp H.: 579

Khak V.A.: 1192

Kidd W.S.F.: 309

Kilias A.: 276, 2075, 2114

Kilias S.P.: 2646

Kiratzi A.: 2135, 2144

Kitsopoulos K.: 2455, 2625

Kokinou E.: 289

Kokkalas S.: 368, 428

Kokkidis N.: 548

Kolaiti E.: 1286

Kolios N.: 2246

Kondopoulou D.: 1888, 1972

Konstantinidi-Syvridi E.: 804

Konstantopoulou G.: 1157, 1619

Kontakiotis G.: 763

Kontogianni V.: 886, 1202

Kontopoulos N.: 558, 643, 654

Koravos G.Ch.: 2193

Koroneos A.: 2606, 2680, 2752

Koskeridou E.: 613

Kosmidis E.: 1812

Kossiaris G.: 939

Kostopoulou V.: 726

Kotsovinos N.: 1074

Kougemitrou I.: 804

Kouki A.: 1169, 1177, 1184

Koukidou I.: 1747

Koukis G.: 1138, 1165, 1210, 1508,
1619

Koukoulis A.: 1457

Koukouvelas I.: 368, 1350

Koulouris S.: 1210

Koumantakis I.: 1590, 1656

Kounis G.D.: 1758, 1767, 1821

Kounis K.G.: 1758, 1767

Kourkouli P.: 1301

Kourkounis S.: 643

Koutsinos S.: 2246

Koutsios A.: 654

Koutsopoulou E.: 2635

Koutsouveli An.: 1418, 1619

Kozireva E.A.: 1341

Kozyreva E.A.: 1192

Kranis H.: 1919

Kritikou S.: 1007

Ktena S.: 1165

Ktenas D.: 548

Kurz W.: 299

Kynigalaki M.: 1202, 1619

Kyriakopoulos K.G.: 309, 663 , 2327, 2361, 2726

Kyrousis I.: 1406

Lagios E.: 344, 2005

Lainas S.: 1138, 1210

Lalechos N.S.: 442

Lalechos S.N.: 442

Lambrakis N.: 1716, 1726, 1878, 2218

Lampropoulou P.: 2465

Laskaridis K.: 2475

Lasocki S.: 2114

Lazaridis A.: 1840, 2383

Lazaris S.: 2390

Lehmann P.: 1831

Leivaditi A.: 1406

Lekkas E.: 1361

Lekkas S.: 1707

Lelli M.: 1840

Lemesios I.: 1878

Leone G.: 886

Leontakianakos G.: 2485

Leptokaropoulos K.M.: 2093

Liakopoulos S.: 1438

Limnios N.: 2200

Locutura J.: 2338

Lois A.: 2183

Loukaidi V.: 737

Loupasakis C.: 1219, 1230, 1465, 1619,1850

Lycourghiotis S.: 1029

Lykakis N.: 2646

Lykoudi E.: 1314, 1406

Lykousis V.: 1046

Magganas A.: 2786 
Makri K.: 169, 999

Makris J.: 32, 357

Makrodimitras G.: 675

Makropoulos K.C.: 216, 2005 , $2015,2084,2104,2163$

Malandraki V.: 1094

Malandrakis E.: 1149

Malegiannaki I.: 1007

Maneta V.: 685

Manoutsoglou E.: 697, 1314, 2492

Maramathas A.: 1777

Marinos P.V.: 1238, 1248, 1259

Marinov S.P.: 2398

Mariolakos I.D.: 92, 821, 829, 1785

Markantonis K.: 1406

Maroukian H.: 409, 418, 506

Marsellos A.E.: 309

Martelli M.: 2327

Matiatos I.: 1792

Mavromatis T.: 1131

Mazzoleni P.: 663

Melfos V.: 845, 948

Mertzanides Y.: 1802, 1812, 1962, 2257

Metaxas A.: 2229, 2236, 2265, 2274

Metaxas Ch.P.: 442

Michail K.: 939

Michailidis K.: 2532, 2657

Midoun M.: 264

Migiros G.: 320

Mirek J.: 2114

Mitropoulos A.: 2257

Mitropoulos D.: 1474

Monaco C.: 400

Moraiti E.: 1267

Moshou A.: 2104

Moumou Ch.: 706

Mountrakis D.M.: 276, 495

Mourtzas N.D.: 453, 1272, 1286

Mpalatsas I.: 2501

Mposkos E.: 2522, 2667

Mwila G.: 1697

Nastos P.T.: 1335

Neuweiler I.: 1831

Nicolaou E.: 939

Nikas K.: 1821

Nikolaidis A.: 989

Nikolakopoulos K.: 1486, 1627 , 1647

Nikolaou N.: 1202, 1393, 1619

Nikolaou P.: 706

Nikolopoulos V.: 829

Nomikou P.: 464, 1056

Novikova T.: 1438

Ntontos P.: 264
Oikonomopoulos I.: 2284

Or D.: 1831

Orlecka-Sikora B.: 2093

Palyvos N.: 829

Pambuku A.: 1777

Panagiotakopoulou O.: 643

Panagiotaras D.: 558

Panagiotopoulos V.: 548

Panagopoulos A.: 1678, 1747

Panagopoulos G.: 2492

Panoussi P.: 634

Pantelaki O.: 697

Papadimitriou E.: 1994, 2200

Papadimitriou E. E.: 1984, 2053, 2064, 2075, 2093, 2114

Papadimitriou P.: 2005, 2015, 2043 , 2084, 2104

Papadopoulos A.: 2680

Papadopoulos G.A.: 1438

Papadopoulou L.: 845

Papadopoulou S.: 548

Papaefthymiou S.: 2465

Papafotiou A.: 1831

Papageorgiou E.: 331, 344

Papakonstantinou K.: 1840

Papamantellos D.: 2465

Papamarinopoulos S.P.: 105

Papanastassiou D.: 1438

Papanicolaou C.: 2294

Papanikolaou D.: 72, 464, 475

Papanikolaou G.: 2236, 2265, 2274

Papanikolaou I.: 320

Papanikolaou M.: 475

Papanikos D.: 939

Papastamatiou D.: 2510

Papastefanou C.: 2680

Papastergios G.: 2373, 2597, 2762

Papathanassiou G.: 486, 1122, $1131,1373,1383$

Papatheodoropoulos P.: 989

Papatheodorou G.: 1018, 1064

Papazachos B.C.: 46

Papazachos C.B.: 46, 495, 1930, 2026, 2064

Papoulia J.: 357

Papoulis D.: 558, 876, 2635

Paradisopoulou P.M.: 2114

Paragios I.: 2597

Paraskevopoulos K.M.: 2752

Parcharidis I.: 1301, 1582

Parpodis K.: 2390

Pasadakis N.: 2294

Pashos P.: 939

Passas N.: 1286

Patronis M.: 2475

Pavlides S.: 169, 486, 1122, 1373,
1383,1607

Pavlides Sp.: 1131

Pavlidou S.: 939

Pavlopoulos A.: 715

Pavlopoulos K.: 1582

Pechlivanidou S.: 706

Perdikatsis V.: 2570

Perissoratis C.: 1035

Perraki M.: 804

Perraki Th.: 2284

Persianis D.: 2692

Petrakaki N.: 2319

Photiades A.: 726, 1495

Pikoulis V.E.: 2183

Pitsonis I.S.: 2193

Plessa A.: 2193

Pliakas F.: 1697

Ploumis P.: 2702

Pomoni-Papaioannou F.: 620, 726, 793

Pomonis P.: 2617, 2712

Pontikes Y.: 856

Popandopoulos G.: 2125

Poulakis N.: 1149

Poulos S.E.: 506

Poutoukis D.: 886, 2383

Poyiadji El.: 1393, 1619

Pratikakis A.: 2562

Pretti S.: 2446, 2588

Psarakis E.Z.: 2183

Psomiadis D.: 886, 958, 1840, 2383

Puglisi D.: 663

Pyliotis I.: 548

Pyrgakis D.: 1138

Pyrgiotis L.: 1619

Raco B.: 1840

Rathossi C.: 856

Rausch R.: 69

Reimann C.: 2350

Rigopoulos I.: 2501, 2617, 2712

Rizzo A.: 2327

Romagnoli G.: 400

Rondoyanni Th.: 379, 1406

Roumelioti Z.: 1438, 2135, 2144

Rousakis G.: 1056

Rozos D.: 1177, 1184, 1219, 1406 , $1465,1590,1637,1656,1850$

Sabatakakis N.: 1138, 1165, 1210, 1619

Sabatakakis P.: 1508

Sakelaris G.: 2786

Sakellariou D.: 1046, 1056

Salminen R.: 2350

Sarris A.: 289

Sboras S.: 486, 1607

Schüth C.: 69 
Schütz C.: 1831

Scordilis E.M.: 46, 2026, 2154

Sdrolia S.: 845

Seeber L.: 2075

Segou M.: 2163

Serelis K.G.: 2390

Serpetsidaki A.: 2174

Siavalas G.: 2218

Sideri D.: 1850

Sifakis A.: 907

Sigalos G.: 737

Sigurdsson H.: 1056

Sikalidis C.: 2373, 2532, 2597, 2762

Skarlatoudis A.A.: 1930

Skarpelis N.: 2417, 2510, 2553

Skianis G.Aim.: 1627, 1647

Skilodimou H.D.: 1572, 1637

Skordas K.: 1858

Smith D.C.: 804

Sofianska E.: 2657

Sokos E.: 989, 2174, 2183

Soldatos T.: 2752

Solomonidou A.: 2726

Sotiropoulos P.: 344

Sotiropoulos S.: 715

Soulios G.: 196

Soulis V.J.: 1094

Soupios P.: 654

Spanos D.: 368

Spanou N.: 1230, 1619

Spassov S.: 1972

Spry P.G.: 2406

Spyridonos E.: 1314, 1785, 2492

Spyropoulos N.: 886

St. Seymour K.: 2406

Stamatakis G.: 2739

Stamatakis M.: 2606, 2739, 2773

Stamatis G.: 1868, 1878

Stamboliadis E.: 697

Stampolidis A.D.: 1907

Stefanova M.: 2398

Stiros S.: 886, 1029

Stivanakis V.: 2465

Stoulos S.: 2680

Stoykova K.: 675

Stratikopoulos K.: 1726

Svana K.: 746

Symeonidis K.: 1286

Syrides G.: 1131

Tagkas Th.: 1149

Tarvainen T.: 2350

Tassiou S.: 1520
Theocharis D.: 821

Theodorou D.: 1335

Theodorou G.: 763

Theodosiou Ir.: 926, 939

Theodosoglou E.: 2752

Thomopoulos Ach.: 1112

Thomopoulos K.: 1064

Tombros S.F.: 2406

Tortorici G.: 400

Tortorici L.: 400

Tougiannidis N.: 2284

Tranos M.D.: 495, 2064

Triantafyllidis S.: 2417

Triantafyllou G.: 2294

Triantaphyllou M.: 475, 602, 634, $715,754,763$,

Trontzios G.: 2657

Tryfonas G.: 1149

Tsagas D.: 1335

Tsaklidis G.M.: 1984, 2200

Tsanakas K.: 418, 506

Tsangaratos P.: 1406, 1590, 1656, 1688

Tsapanos T.M.: 2193

Tsaparas N.: 620

Tselentis G-A.: 2174

Tselepides V.: 379

Tsiambaos G.: 183, 1104, 1259

Tsikouras B.: 876, 2501, 2540, 2617,2712

Tsimas S.: 2485

Tsipoura-Vlachou M.: 663

Tsirambides A.: 2606, 2762

Tsirigotis N.: 1149

Tsobanoglou C.: 1812

Tsokas G.N.: 1907

Tsolakis E.: 763

Tsolis-Katagas P.: 856, 2635

Tsombos P.: 1438, 1447, 1486, $1528,1539,1547,1539,1548$, 1559

Tsoukala E.: 958

Tsourlos P.: 1962

Tzamos E.: 2762

Tzanaki I.: 289

Tzanis A.: 344, 1919, 1941

Tzavidopoulos I.: 886

Tzevelekou Th.: 2465

Tziritis E.: 1858

Tzortzaki E.: 613

Vafidis A.: 1802

Vagenas N.: 1165

Vagenas S.: 1210
Vagiotou E.: 1149

Vaiopoulos D.: 1627, 1647

Vakalas I.: 675, 697

Vako E.: 1777

Valera P.: 2446, 2558

Valiakos I.: 965

Valkaniotis S.: 486, 1383

Vamvakaris D.: 495

Van Cappellen P.: 2310

Varaggouli E.: 1074

Vargemezis G.: 1953, 1962

Varnavas S.: 234

Varvarousis G.: 2229, 2265

Vasilatos Ch.: 2773

Vassiliades E.: 1520

Vassiliou E.: 1688

Vassilopoulou S.: 516

Vaxevanopoulos M.: 948

Vitsas T.: 989

Vlachopoulos I.: 1165

Vlachou-Tsipoura M.: 2773

Vogiatzis D.: 2762

Vontobel P.: 1831

Votsi I.: 2200

Voudouris K.: 1678

Voudouris P.: 685, 845, 2786

Vougioukalakis G.: 939

Voulgaris N.: 2163

Vouvalidis K.: 706, 1122

Vrettos K.: 2236

Vythoulkas N.K.: 2193

Wölfler A.: 299

Xeidakis G.: 1074

Xypolias P.: 368, 387

Zagana E.: 1726, 1878

Zambetakis-Lekkas A.: 773

Zananiri I.: 1474, 1539, 1549, 1972

Zanchetta G.: 886

Zelilidis A.: 643, 675, 697, 793

Zerefos C.S.: 2

Zervakou A.D.: 1528, 1539, 1549, 1559

Zevgitis T.: 989

Ziannos V.: 1812

Zidianakis G.: 781

Zisi N.: 958, 1840, 2383

Zorba T.: 2752

Zoumpoulis E.: 793

Zouridakis N.: 1792

Zouros N.: 159, 896, 965

Zygouri V.: 527 